\section{PERMISSIVE HYPERCAPNIA RESULTS IN A DECREASED FUNCTIONAL VESSEL DENSITY IN THE SKIN OF EXTREMELY LOW BIRTH WEIGHT INFANTS}

\author{
K. Schottmayer ${ }^{1}$, O. Genzel-Boroviczeny ${ }^{1}$, \\ S. Henneberger ${ }^{1}, \mathrm{U}$. Thome ${ }^{2}$ \\ ${ }^{1}$ Neonatology, LMU, Munich, ${ }^{2}$ Neonatology, \\ University Leipzig, Leipzig, Germany
}

Background: Due to the increasing number of extremely low birth weight infants (ELBI) long term ventilation and its aftermath has become an important issue. One of the newer concepts for gentle ventilation is permissive hypercapnia. We were interested in the effects of the higher $\mathrm{pCO}_{2}-$ levels on the microcirculation of ELBIs.

Methods: Data were collected from 12 infants, who were randomized either to treatment with permissive hypercapnia or normocapnia. Inclusion criteria were a birth weight between $400 \mathrm{~g}$ and $1000 \mathrm{~g}$, a gestational age from $23^{\text {rd }}$ to $28^{\text {th }}+6$ weeks, intubation during the first 24 hours of life and no malformations. The $\mathrm{pCO}_{2}$ target range was increased stepwise and was $15 \mathrm{mmHg}$ higher in the intervention group. Skin microvascular parameters were assessed noninvasively with SDF on the right arm every $24 \mathrm{~h}$ during the first week of life and on the $14^{\text {th }}$ day.

Result: $\mathrm{pCO}_{2}$ (AUC: $639 \pm 62$ vs $744 \pm 62$ ) differed significantly between the two groups $(p=0,04)$. Functional vessel density (FVD) was significantly lower in the intervention group on the $14^{\text {th }}$ day of life $\left(395 \pm 43\right.$ vs $\left.281 \pm 67 \mathrm{~cm} / \mathrm{cm}^{2} ; \mathrm{p}=0.04\right)$. The proportion of small vessels increased in the control group whereas they decreased slightly in the intervention group, but did not reach stat. sig. Increasing target $\mathrm{pCO}_{2}$ lead to a temporary hyperdynamic flow in both groups.

Conclusions: $\mathrm{pCO}_{2}$-levels influence significantly the microcirculation in preterm infants. Elevation of $\mathrm{pCO}_{2}$-levels leads to a decrease in FVD, presumably due to shunting and vasoconstriction and might cause temporarily hyperdynamic flow.

\section{EXCESSIVELY HIGH BILIRUBIN AND EXCHANGE TRANSFUSION IN VERY LOW BIRTH WEIGHT INFANTS}

\author{
J. Kuint ${ }^{1}$, A. Maayan-Metzger ${ }^{2}$, V. Boyko², \\ L. Lerner-Geva ${ }^{2}$, B. Reichman² \\ ${ }^{1}$ Neonatology, ${ }^{2}$ Sheba Medical Center, \\ Ramat Gan, Israel
}

Objective: To evaluate the distribution of peak serum bilirubin levels in very low birth weight (VLBW) infants and the performance of exchange transfusion in infants with excessively high bilirubin levels.

Methods: A population based observational study using data collected by the Israel National VLBW Infant Database. The study sample comprised 13,499 infants of 24 to 33 weeks gestation. Two definitions of excessively high peak bilirubin levels which might be considered as threshold levels for performance of exchange transfusion were used. Firstly, a bilirubin level of $\geq 15 \mathrm{mg} / \mathrm{dL}$ for all infants (PSB-15), and secondly, incremental bilirubin levels ranging from $12-17 \mathrm{mg} / \mathrm{dL}$ according to gestational age (PSB-GA). Multivariable logistic regression analyses were performed to examine the factors associated with performance of exchange transfusion.

Results: Four hundreds sixty eight $(3.5 \%)$ and 1035 infants $(7.7 \%)$ infants in the PSB-15 and in the PSB-GA groups respectively had peak serum bilirubin levels above thresholds for exchange transfusion. Exchange transfusion's were performed in only $66(14.1 \%)$ of these infants in the PSB-15 group and 91 (8.8\%) in the PSB-GA group. In both groups performance of exchange transfusion was significantly associated with peak serum bilirubin levels, with an odds ratio of 1.38 for each $\mathrm{mg} / \mathrm{dL}$ increase above the threshold level.

Conclusion: Exchange transfusion was performed in only $9-14 \%$ of VLBW infants with excessively high bilirubin levels. This may be related to an absence of definitive guidelines or the possible belief that the risks of exchange transfusion outweigh the potential risk of bilirubin induced neurological injuries. 